Quebec Cooperative Study

of Friedreich's Ataxia

\title{
The Cardiomyopathy of Friedreich's Ataxia Morphological Observations in 3 Cases
}

\author{
J.B. LAMARCHE, M. CÓTÉ, B. LEMIEUX
}

SUMMARY: In the light of the recent finding of deposits of calcium salts and iron in myocardial cells in one case of Friedreich's ataxia, we have made a detailed morphological study of 3 new cases of this cardiomyopathy. Calcium deposits were not found in the muscle fibers but lipofuscin granules and deposits of iron were observed in our 3 cases. In addition to the usual findings of inter-

RESUME: Suite à l'identification récente de sels de calcium et de fer dans les cellules musculaires cardiaques d'un cas d'ataxie de Friedreich. nous avons fait une étude morphologique approfondie de 3 nouvelles observations de cette cardiomyopathie.

Nous n'avons pas trouvé de dépôts de calcium mais nous avons observé des dépôts de fer et des granules de lipofuscin dans les fibres cardiaques de nos 3 cas.

En plus de la fibrose interstitielle, de stitial fibrosis, hypertrophy and degeneration of myocardial fibers, foci of segmental active muscle necrosis were constantly present. There is a possibility that Friedreich's ataxia could be a neurocardiac degenerative disease with a membrane defect which could be related to defective metabolism of vitamin E or other micronutrients.

l'hypertrophie et la dégénérescence des fibres myocardiaques observées habituellement dans cette cardiomyopathie, nous avons retrouvé de façon constante des foyers de nécrose musculaire segmentaire active. Il est possible que l'ataxie de Friedreich soit une maladie dégénérative neuro-cardiaque associée à un défaut membranaire qui pourrait être lié à un trouble métabolique de la vitamine $E$ ou autres micro-nutriments.
From the Centre Hospitalier Universitaire de Sherbrooke

Reprint requests for the complete supplement on Friedreich's Ataxia (phase three) to: Dr. André Barbeau, Clinical Research Institute of Montreal, 110 Pine Avenue West, Montreal, Quebec, Canada, H2W IR7.
The pathological changes in the heart in Friedreich's ataxia are well known and have been recently reviewed by Sanchez et al. (1976). They consist mainly of a cardiomegaly with ventricular hypertrophy and are characterized histologically by myocardial fibrosis, hypertrophy and degeneration of the cardiac muscle fibers. Over the past 10 years, an increasing number of the hypertrophic form of cardiomyopathy has been detected clinically in patients with Friedreich's ataxia and it has been suggested by some authors (Smith et al., 1977) that hypertrophic cardiomyopathy might be specific to classical Friedreich's ataxia.

Myocardial alterations not previously described in the literature have been reported, recently, in one case of Friedreich's ataxia. Sanchez et al. (1976) found granular deposits of calcium salts, and of iron in degenerated cardiac muscle cells; they interpreted these findings as a probable dystrophic metabolic change secondary to cellular degeneration and fibrosis and being possibly related to the disease process.

In an attempt to further define the histophathological changes in the heart in Friedreich's ataxia, especially in the light of the recent finding of mineral deposits in myocardial cells, post-mortem material was studied from 3 patients with neuropathologically documented classical Friedreich's ataxia.

\section{PATIENTS AND METHODS}

Anatomical data concerning the cardiovascular system were obtained from the necropsy protocol in case 1 and 2. Paraffin blocks or/and formalin fixed tissue from the hearts and all other viscera were available in the 3 cases. Several sections of both ventricles were available in case 1 . Numerous 
heart samples were obtained in case 2 but their topographical site of origin was unspecified. In case 3, the complete intact but formalin fixed heart was available and it was possible to make a detailed gross observation together with a representative sampling of all major cardiac structures including the coronary arteries, the septum and the conduction system.

In cases $I$ and 3 , fresh cardiac tissue obtained approximately 2 hours after death was processed for examination by electron microscopy. Small fragments of myocardium were fixed in Scollidine-buffered $4 \%$ para-formaldehyde, $\mathrm{pH} 7.4$ and post fixed in $2 \%$ osmium tetroxide buffered to $\mathrm{pH} 7.4$ with S-collidine. After dehydration the tissues were embedded in epon resin. Thin sections were examined in a Philips 300 electron microscope after staining with uranyl-acetate and lead citrate.

All sections for light microscopic examination were stained with hematoxylin-eosin. The following special stains were utilized on cardiac tissue and appropriate control material whenever indicated: Masson's trichrome for collagen, Verhoeff's for elastic, PAS with or without diastase, Von Kossa for calcium, Sudan black B, Ziehl-Neelsen for acid fast material and Prussian blue for iron.

Unstained deparaffinized sections from the myocardium were examined under polarized and fluorescent light.
Samples from all viscera were also stained for iron and calcium.

\section{Case 1: Clinical summaries (L.F.) (A-58-77)}

This patient was a 18-year-old white Caucasian female with Friedreich's ataxia which first manifested at the age of 2. Two siblings out of eight were also afflicted. She had been confined to a wheel-chair since the age of 10 . At age 6 , the electrocardiogram (E.C.G.) showed left ventricular hypertrophy and cardiac catheterization was compatible with a non-obstructive hypertrophic cardiomyopathy. Both the right and left ventricular end-diastolic pressures were elevated at 16 and 22 $\mathrm{mm} / \mathrm{Hg}$ respectively. During the following years, she complained of intermittent palpitations which were never documented by E.C.G. She did not experience chest pains or shortness of breath and she was not susceptible to respiratory infections.

One month prior to death, the blood pressure was $100 / 65$, heart rate regular $80 /$ minute, no jugular venous distension was noted. There was a marked right dorsal scoliosis which was found to be at $45^{\circ}$ on X-Rays. On auscultation, the lungs were clear and the heart sounds normal with no murmur or gallop rhythm. Chest X-Ray showed no evidence of cardiomegaly or pulmonary hypertension. The E.C.G. showed diffuse STT changes with questionable right ventricular hyper- trophy and right axis deviation. The vectocardiogram however, was compatible with a type $B$ right ventricular hypertrophy pattern. The left ventricular diastolic and systolic diameters by M-mode echocardiography were small, 30 and 20 respectively, but the septal and free posterior wall thickness were within normal limits at $10 \mathrm{~mm}$.

Two days prior to death, she developed nausea, vomiting of blood streaked material and progressively prolonged apneic spells leading to her demise. There was no clinical evidence of congestive heart failure or tachyarhythmias.

Summary of pathological findings:

In the lungs, multiple peripheral emboli and hemorrhagic infarcts were observed. A superficially ulcerative gastro-oesophagitis with a major gastro-intestinal hemorrhage was found. Chronic passive congestion of the liver was noted.

\section{Cardiovascular system:}

The heart weighted $320 \mathrm{gm}$. The thickness of the left ventricle was 1.2 $\mathrm{cm}$ and the right $.4 \mathrm{~cm}$. A slight dilatation of the right cardiac chambers was noted. There was slight arteriosclerosis of the aorta. Only samples of both ventricles were available. In all sections, the microscopic examination showed interstitial diffuse fibrosis, moderate in the left ventricle and slight in the right ventricular wall. There was also marked hypertrophy of the muscular

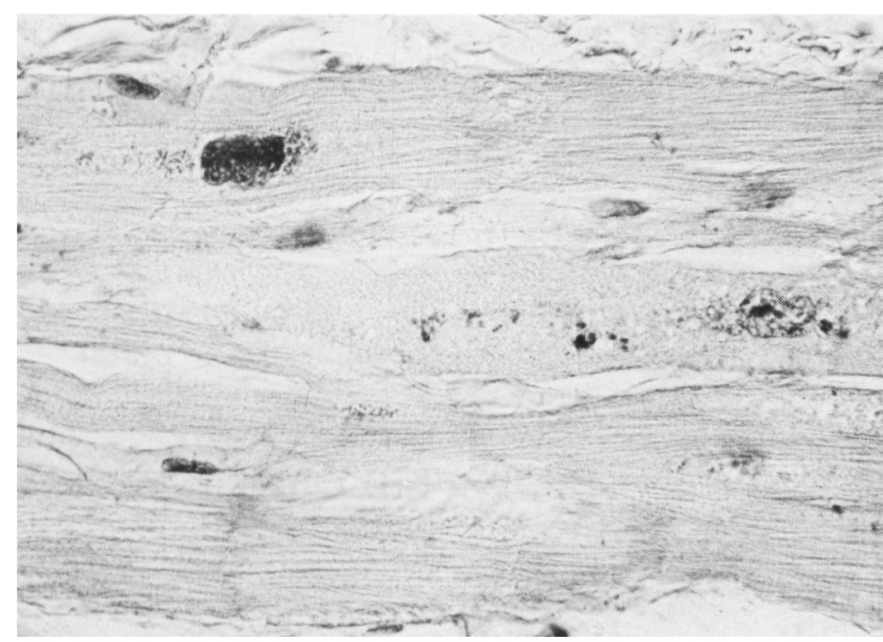

Figure I Case I - Hypertrophied myocardial fibers with large bizarre hyperchromatic nuclei. Note the peripheral distribution of the myofibrils and the granular appearance of the cytoplasm. Lipofuscin granules are present mainly in the paranuclear area of the nuclei. (PAS X 530)

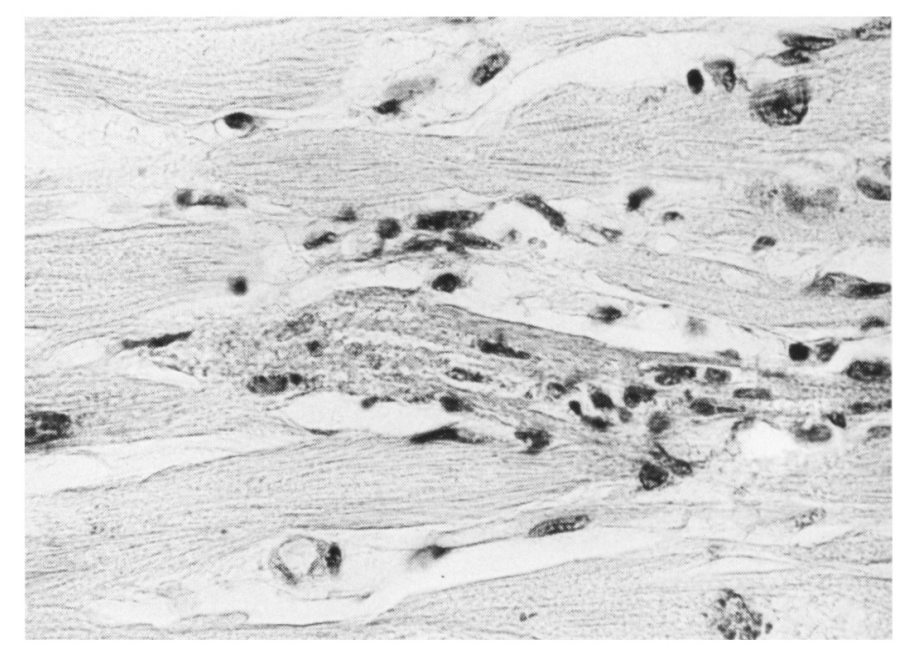

Figure 2 Case 1 - Segment of a myocardial fiber showing early necrosis associated with a few mononuclear cells. (PAS X 530) 
fibers with large pleomorphic hyperchromatic nuclei containing an occasional eosinophilic nucleoliform inclusion. In several fibers, the cytoplasm, which frequently contained yellowbrown pigment, was finely granular and/or vacuolated, the longitudinal myofibrils occupying a peripheral location. (Figure 1) No disorientation of myocardial fibers was observed. A small number of chronic inflammatory cells was present focally in the interstitium. There were individual and rare groups of muscle fibers undergoing acute necrotic changes with occasional polymorphonuclear leucocytes and frequent mononuclear cells. (Figure 2 and 3) Slight to moderate focal intramural arteriolar thicknening was also observed.

The yellow-brown granules were autofluorescent, acid fast positive and stained with the Sudan black. These granules were also slightly PAS positive in some areas. No calcium was found, but dense intracytoplasmic deposits of iron particles were detected in a small number of cardiac fibers in the paranuclear areas. Ultrastructural studies showed several membrane bound osmiophilic particles characteristic of lipofuscin granules. (Figure 4) No calcium or iron was detected in the parenchyma of the other viscera.

Case 2: (R.T.) (A-25-72)

This was a 22-year-old white Cau- casian male suffering from Friedreich's ataxia and confined to a wheel chair since the age of 12 . Two sisters and two brothers were also afflicted with the same disease and the eldest died at the age of 25 . The patient was intellectually alert and did not complain of excessive shortness of breath or palpitation. One year prior to death, however, his heart rate was found to be intermittently irregular but no medication was prescribed. Four days before his last admission at another hospital, he developed a febrile illness with a fever varying between 101 to $102.5^{\circ} \mathrm{F}$. and a non-productive cough. Two days later, the cough and fever persisted and he also developed nausea and abundant vomiting with no evidence of gastro-intestinal diathesis. Physical examination on admission two days later revealed a conscious but disoriented tachypneic and pale patient. His heart rate was 150 to $180 /$ minute, irregular, his blood pressure $80 \mathrm{~mm} / \mathrm{Hg}$ systolic. There was no jugular venous distension. The thoracic cage was severely deformed by a marked right dorsal scoliosis. Auscultation or gallop sound was audible. There was no peripheral edema. Severe dysarthria, muscle weakness and atrophy was evident. The chest XRay revealed a marked roto-scoliosis of the dorsal spine which rendered the cardiac shadow difficult to evaluate. There was no clear evidence of parenchymal infiltrates or pulmonary vascular congestion. The E.C.G. showed atrial fibrillation with a rapid ventricular rate of $230 /$ minute with right bundle branch block and right superior axis deviation. Intermittent left bundle branch block or ventricular tachycardia was also observed. He died a few hours after admission in a persistent state of shock and anuria.

\section{Summary of autopsy findings:}

Early bronchopneumonia in the lower lobes was observed. Marked chronic passive congestion with central necrosis was noted in the liver. Vacuolar changes consistent with osmotic nephrosis were observed in the tubules of both kidneys.

\section{Cardiovascular system:}

The heart weighted $425 \mathrm{gm}$. The thickness of the left ventricle was 1.5 $\mathrm{cm}$. and the right $.4 \mathrm{~cm}$. There was a slight left ventricular hypertrophy. No arteriosclerotic changes were observed in the coronary arteries or the aorta.

A great number of paraffin blocks of unspecified sites of origin were available. Histopathological findings included a mild focal chronic inflammatory infiltrate and interstitial fibrosis which was generally slight but moderate in few areas. Moderate hypertrophy of the myocardial fibers was observed together with large pleomorphic and hyperchromatic nuclei con-

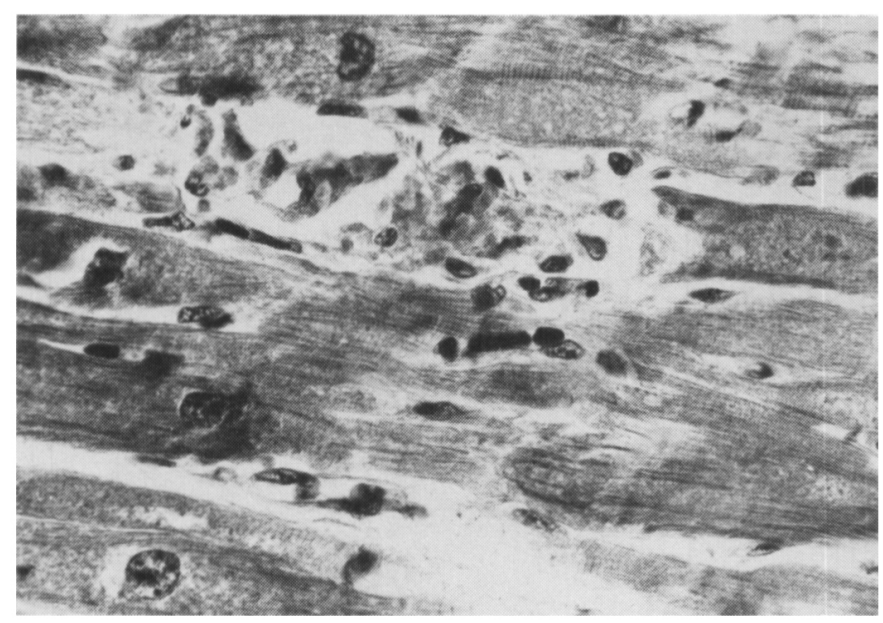

Figure 3 Case I - Segment of myocardial fiber showing a slightly more advanced stage of necrosis. There is fragmentation of the necrotic focus with a mononuclear inflammatory infiltrate. Note also the bizarre hyperchromatic nuclei, the granular aspect of the cytoplasm and the peripheral distribution of the myofibrils. (Masson's trichrome X 530)

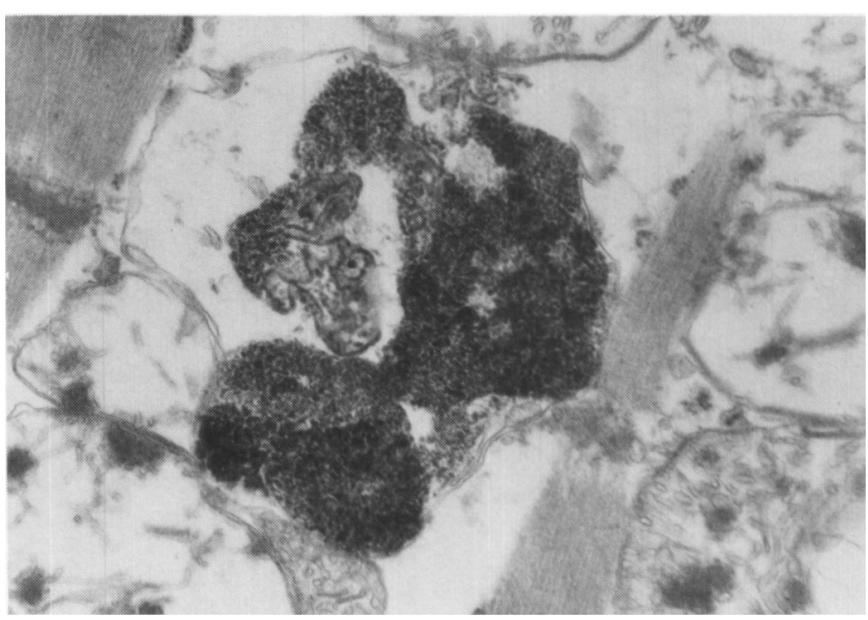

Figure 4 Case 1 - Electron micrograph of lipofuscin granules partially surrounded by a single or possibly a double membrane (X 42630) 


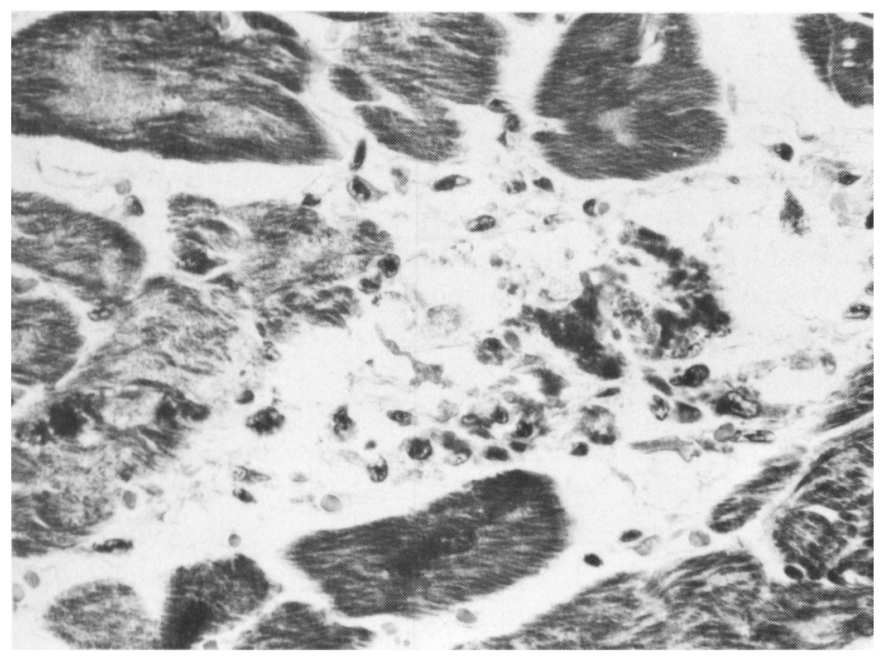

Figure 5 Case 2 - Segmental necrosis with desintegration of the myocardial fiber and mononuclear cell infiltrate. (Masson's trichrome X 530)

taining occasional nucleoliform eosinophilic intranuclear inclusions. No myocardial fiber disarray was noted. There was muscular vacuolization, a frequent peripheral myofibrillary distribution and a finely granular appearance of the cytoplasm. Occasional segmental necrosis of myocardial muscle cell was noted. (Figure 5) Thickening of the small intramural coronary vessels was generally slight, but in some areas, there was moderate subintimal fibrosis. Von Kossa stain for calcium was negative. The Prussian blue stain for iron demonstrated dense accumulations of positive particles in the paranuclear zones of the fibers (Figure 6) and occasionally within macrophages near or inside necrotic fibers. The number of myocardial fibers with iron deposits was greater than in case 1. Lipofuscin pigment was also abundant in several fibers as seen in the previous case.

No iron or calcium was identified in the parenchyma of other viscera.

\section{Case 3: (G.H.) (A-257-76)}

This was a 28 -year-old white Caucasian male suffering from Friedreich's ataxia since the age of 5 . He has been confined to a wheel chair since age 13 . One year prior to his death, he presented with intermittent atrial fibrillation associated with dyspnea and nausea. Physical examination at that time revealed an irregular heart rate at 150 /minute, blood pressure 90 $\mathrm{mg} / \mathrm{Hg}$ systolic and no jugular venous distension. There was a marked left dorsal scoliotic deformation of the thorax. The lungs were clear and no heart murmurs or gallop rhythm were audible. The E.C.G. showed atrial fibrillation with a rapid ventricular rate and diffuse ST-T changes. The right and left ventricular diameters were within normal limits $(15 \mathrm{~mm}$ and $45 / 35 \mathrm{~mm})$ by echocardiography which also revealed a septal and posterior wall of normal thickness $(8$ $\mathrm{mm})$. Chest X-Ray showed an enlarged cardiac silhouette, pulmonary infiltrates and confirmed the scoliosis.

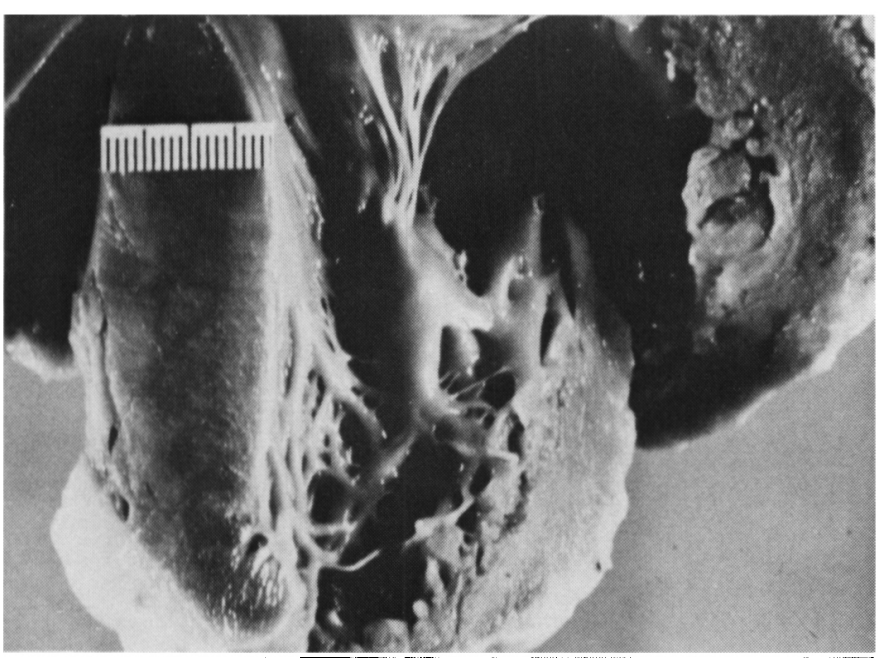

Figure 7 Case 3 - Heart showing the hypertrophy of the septum (ruler) which measures $2 \mathrm{~cm}$ in thickness. The left ventricular free wall, to the right side of the septum, is $1.4 \mathrm{~cm}$ thick.

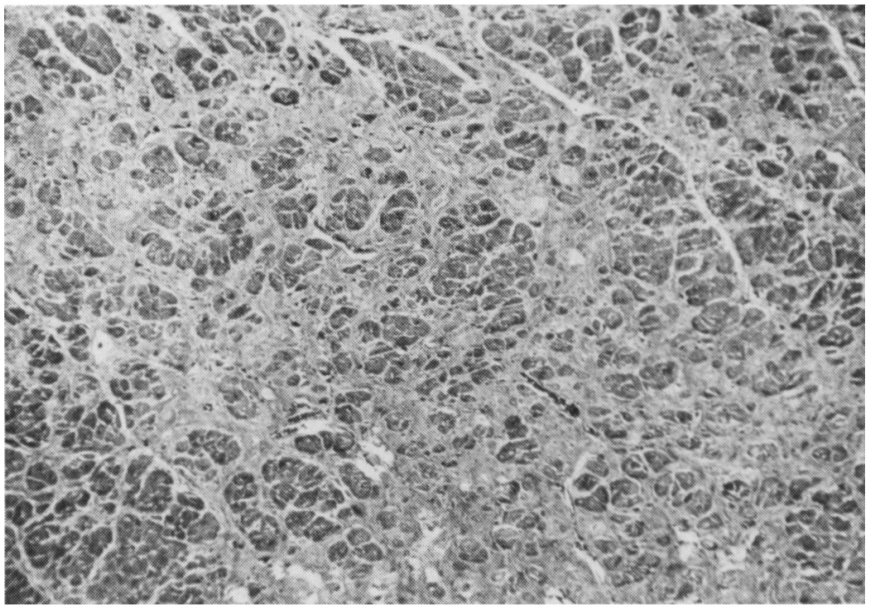

Figure 8 Case 3 - Left ventricle showing marked interstitial fibrosis and the hypertrophy of the myocardial fibers. (Masson's trichrome $\mathrm{X} 33$ ) 


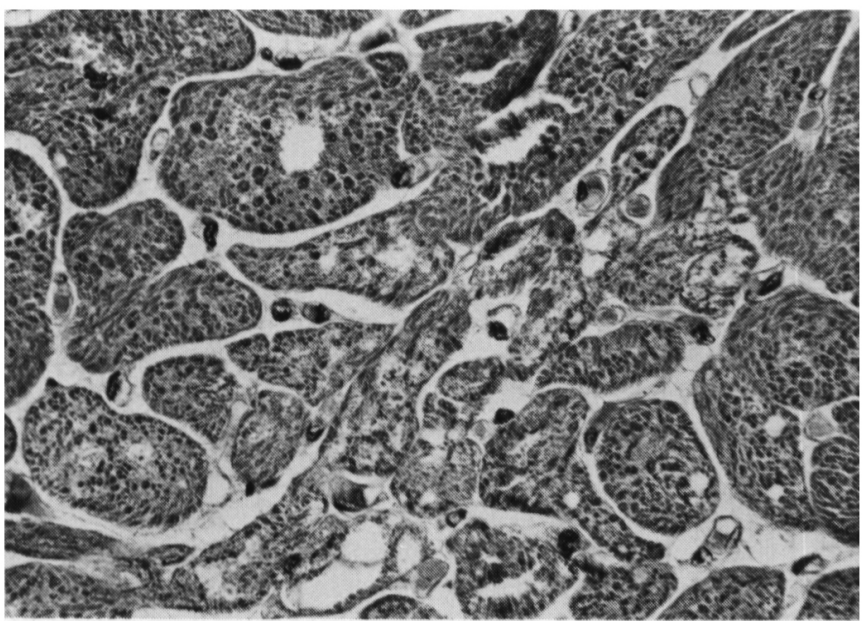

Figure 9 Case 3 - Hypertrophied myocardial fibers with clearly seen individual myofibrils of variable diameters. Note the vacuolar changes in several fibers. (Masson's trichrome X 530)

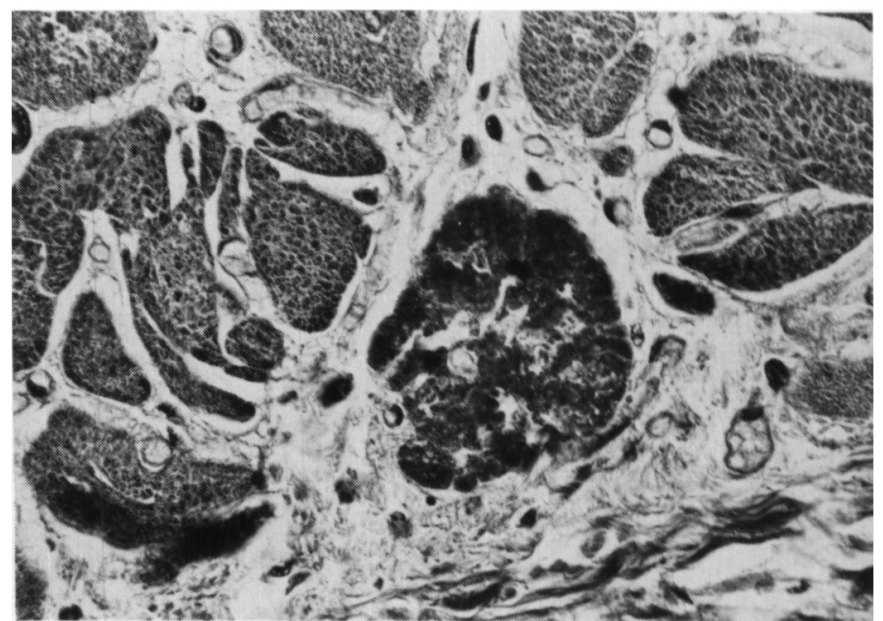

Figure 10 Case 3 - Cross section of hypertrophied myocardial fiber showing early necrosis. (Masson's trichrome X 530)
The right heart catheterization revealed a moderately elevated right end-diastolic pressure at $16 \mathrm{~mm} / \mathrm{Hg}$ and a slightly raised pulmonary capillary wedge pressure at $18 \mathrm{~mm} / \mathrm{Hg}$. A discrete $13 \mathrm{~mm} / \mathrm{Hg}$ right intraventricular systolic pressure gradient was also documented. Subsequently, he remained in atrial fibrillation and terminally presented with respiratory distress characterized by episodes of apnea and respiratory pump failure without evidence of significant congestive heart failure. He also complained of abdominal pains but there was no clinical evidence of gastro-intestinal bleeding.

Summary of autopsy findings:

A non-caseating granulomatous pro- cess consistent with sarcoidosis was observed in the lungs, spleen, paratracheal and hilar lymph nodes, liver and myocardium. Multiple foci of atelectasis were observed in both lungs. Erosive gastritis was also found.

\section{Cardiovascular system:}

There was minimal abdominal aortic atherosclerosis with a slightly hypoplastic aortic arch. The heart weighted $440 \mathrm{gm}$. The thickness of the left anterior ventricular free wall was $1.4 \mathrm{~cm}$., the left posterior free wall; 1.3 $\mathrm{cm}$, the right ventricle; $.3 \mathrm{~cm}$. and the interventricular septum; $2 \mathrm{~cm}$. (Figure 7) The diameter of the valves was: pulmonary; $5.5 \mathrm{~cm}$., tricuspid; $9.5 \mathrm{~cm}$., aortic; $5.5 \mathrm{~cm}$. and mitral; $9.0 \mathrm{~cm}$. The ventricular septum was thicker than the thickest portion of the left ventricular free wall. There was also a slight hypertrophy and dilatation of the right ventricle. Examination of the coronary arteries revealed slight to moderate arteriosclerosis with luminal narrowing varying from 25 to $40 \%$.

The tissue sampling was extensive and included the conduction system and the large coronary arteries. In all sections of the heart, the microscopic examination revealed interstitial fibrosis generally moderate to marked. (Figure 8). The fibrosis was minimal in the auricular appendages and in the conduction system. Occasional noncaseating granulomata were observed, but they were scarse considering the

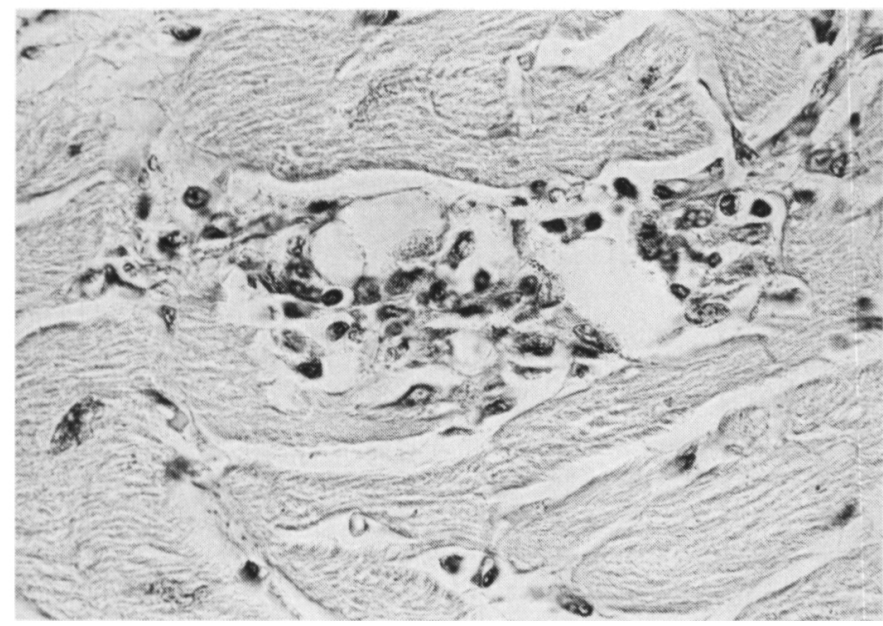

Figure II Case 3 - Advanced stage of segmental necrosis with almost total dissolution of the fiber and dense mononuclear inflammation infiltrate. (PAS X 530)

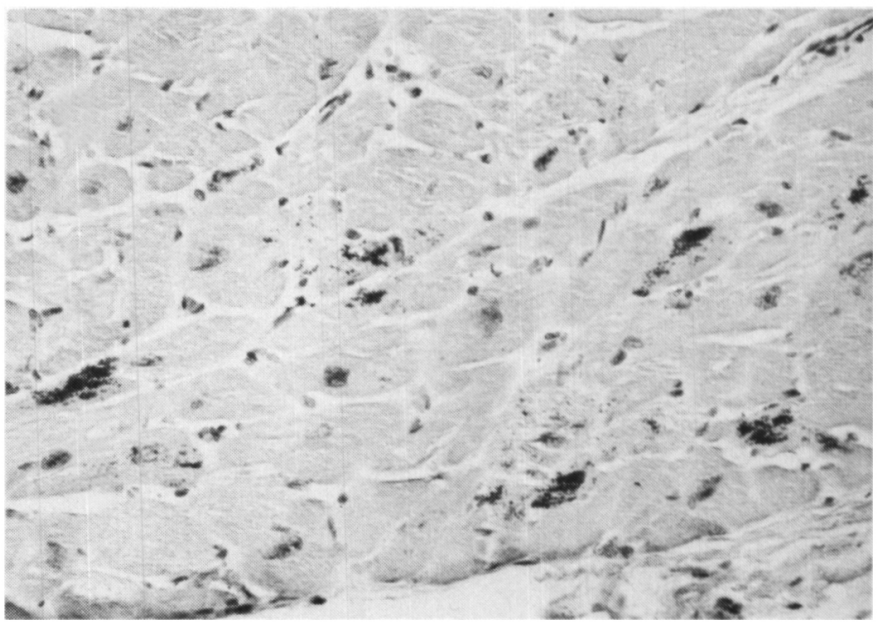

Figure 12 Case 3 - Dense intracytoplasmic deposits of iron are observed in several cardiac fibers. (Prussian blue $X$ 212) 
extent of the sampling. In all sections, there were muscle changes characterized by hypertrophy, a granular or vacuolated appearance of the cytoplasm and a peripheral distribution of the myofibrils. (Figure 9) No muscle fiber disarray was seen. Pleomorphic and hyperchromatic nuclei containing occasional nucleoliform eosinophilic inclusion bodies were also observed. Segmental necrosis of individual muscle fibers with or without phagocytosis was occasionally seen. (Figure 10 and 11) There was also a focal chronic inflammatory infiltrate in the interstitium. The intramural small coronary vessels showed slight to moderate focal thickening. The large coronary vessels showed slight atheromatous changes.

No calcium was found but predominantly paranuclear intracytoplasmic iron was identified in several cardiac fibers. (Figure 12) These iron deposits were sightly more numerous than in case 2 and were observed mainly in the left ventricle, in the septum and the right auricle. Ironladen macrophages were sometimes identifed within necrotic myocardial fibers. Paranuclear intracytoplasmic lipofuscin pigment was also observed in numerous cardiac fibres. (Figure 13)

\section{DISCUSSION}

The gross and histopathological changes observed in these 3 cases are generally in keeping with those reported in the literature. In addition to the fibrosis and the chronic inflammatory infiltrate which were present in varying proportions, there was also a striking hypertrophy of the myocardial fibers together with constant peculiar cytoplasmic and nuclear alterations previously well described in 1946 by Russell. The nuclei were always large and irregular in shape with an increased amount of chromatin. These nuclear changes have been observed in myocardial hypertrophy (Bove, 1974), in obstructive and idiopathic cardiomyophathies (Heggtveit and Nadkarni, 1971) and presumedly reflect polyploidy (Sandritter and Scomazzoni, 1964). Small intranuclear eosinophilic bodies mimicking a nucleolus and probably representing an invagination of the cytoplasm were occasionally noted. We have not found any explanations for this finding. The cytoplasmic vacuolar and granular alterations bear some resemblance to those observed in human disorders of skeletal muscle of various etiologies (Adams et al., 1962). Vacuolar degeneration of both striated muscles

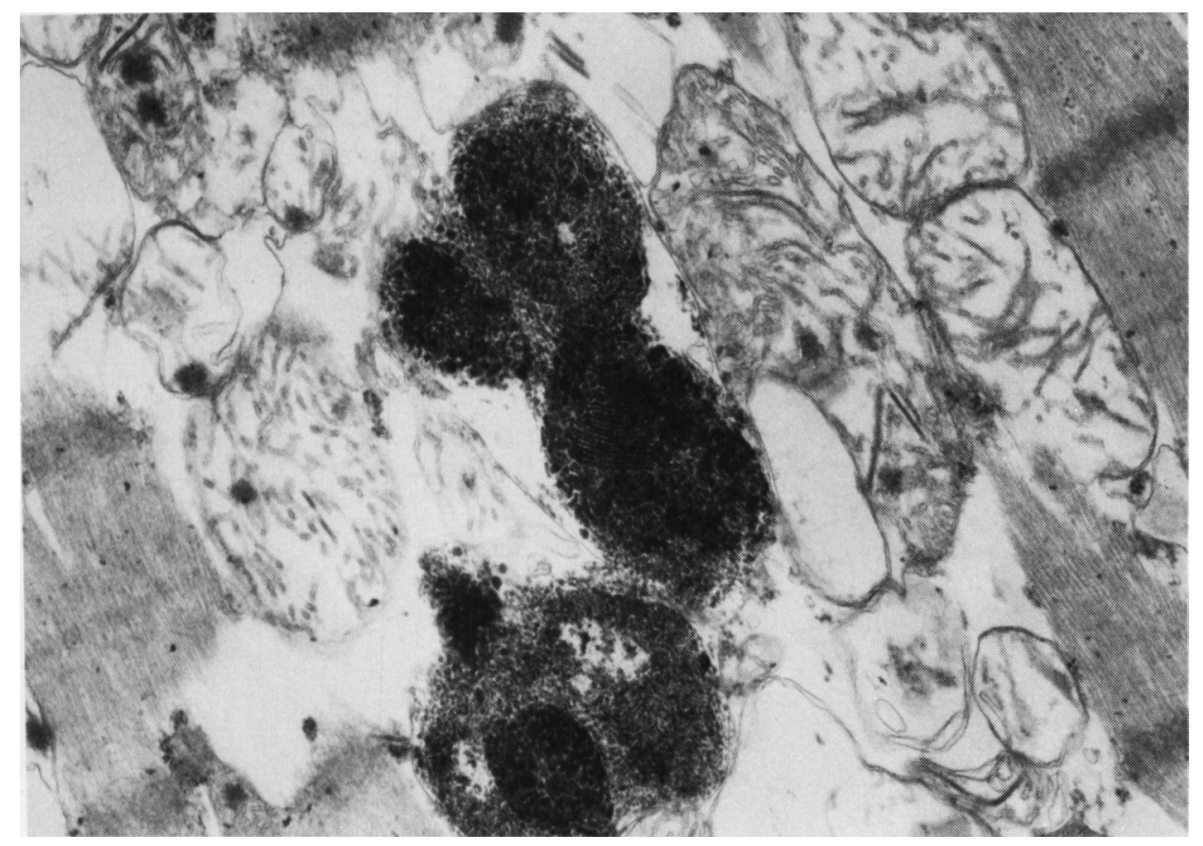

Figure 13 (Case 3) - Electron micrograph of lipofuscin particles surrounded by a single or possibly a double membrane. Note the fingerprint pattern in one granule. (X 34884) and myocardial fibers has been observed in a human neuromuscular disorder of undetermined nature (Morales, 1974).

In all 3 cases, we found varying amounts of intracytoplasmic yellowbrown pigmented granules mainly in the region of the nuclei. On the basis of their autofluorescence, their ultrastructural morphology (Malkoff and Strehler, 1963) and their staining characteristics (Nishioka et al., 1968) these granular deposits are lipofuscin. The presence of lipochrome pigments in the myocardium of patients with Friedreich's ataxia has been occasionally mentioned in a few observations (Russel, 1946). As no quantitative studies were made, our finding could merely represent normal accumulation of lipochrome pigment for the age. Strehler et al., (1959) have shown that these pigments start to accumulate in the human myocardium around age 10 and their concentration is age dependent increasing progressively in a linear fashion at a rate of approximately one-third of a percent of heart volume/decade. Occasionally, however, an increased accumulation of cardiac lipofuscin has been reported in relatively young persons especially in conditions associated with inanition and also in vitamin $E$ deficient animals (Koobs et al., 1978). It has been suggested by Koobs et al. (1978) that the transformation of mitochondria into lipofuscin granules could occur as the result of peroxidative destruction of poly-unsaturated fats in lipid membranes. Nuclear damage produced by malonaldehyde, a byproduct of lipid peroxidation, could reduce the capacity for protein synthesis and thus limit mitochondrial and contractile fibre replacement.

Our studies did not reveal any myocardial calcium deposits in these 3 cases as was originally reported by Sanchez et al., (1976) in one observation. Calcification of cardiac muscle fibers has been observed in a great variety of human and experimental conditions (Gore and Arons 1949, Reichenbach and Benditt, 1969) and appears to be, in most instances, a nonspecific alteration related to myocardial cell degeneration and necrosis.

We have, however, identified in all 3 cases deposits of iron in several well 
preserved myocardial fibers and in a few macrophages within necrotic fibers. This finding which was reported for the first time by Sanchez et al., (1976) remains unexplained. The absence of iron in the parenchymal cells of other viscera or in the reticuloendothelial system in our cases argue against a generalized iron storage disease. Buja and Roberts (1971) have shown that, when iron deposits are found in the myocardium in hemochromatosis and hemosiderosis, they are always present in other viscera particularly in liver, spleen, pancreas and lymph nodes. In their study, these authors found cardiac hypertrophy and dilatation, degeneration of muscle fibers, dystrophic calcification and myocardial fibrosis in association with intracardiac iron deposits. The myocardial alterations were localized in areas of maximum iron deposition. In our cases, there was no correlation between the amount of iron deposits and the degree of fibrosis and/or necrosis although iron laden macrophages in areas of muscle necrosis were occasionally observed. The consistent association of iron with intact myocardial fibers is against a dystrophic type of metabolic change. There is a possibility that the iron might be present within some lipofuscin granules as previously reported by a minority of observers (Porta and Hartroft, 1969). In our control material, we found lipofuscin granules within myocardial fibers but no iron was detected.

Foci of myocardial necrosis have been exceptionally observed in Friedreich's ataxia. Russell (1946) reported one observation out of 4 with necrosis of muscle fibers and Hewer (1964) described similar changes in one case out of 16. In our 3 cases, the presence of focal myocardial necrosis was a remarkable finding. These necrotic foci were usually segmental and generally associated with an inflammatory reaction consisting predominantly of mononuclear cells including some macrophages. Muscle fibers at different stages of fragmentation could be seen. These findings, which indicate an actively progressing process, bear some striking similarities to the segmental necrosis observed in primary diseases of skeletal muscle especially in the dystrophies (Pearce and Walton, 1962) and the inflammatory myopathies (Walton and Hudgson, 1977). The vascular narrowing observed in the main coronary arteries and the intramural arterioles was never marked and was out of proportion to the extent of the fibrosis. Furthermore, no relationship between the vascular narrowing and the topography of the necrotic foci could be established.

In case 3 , there was a grossly disproportional hypertrophy of the ventricular septum consistent with an hypertrophic form of cardiomyopathy (Roberts et al., 1974). However, the characteristic histological pattern of myocardial fiber disarray reported in this form of cardiac disease was not convincingly observed in our case. Furthermore, hypertrophic cardiomyopathy is not usually associated with necrosis or with an inflammatory infiltrate.

Regarding the granulomatous process consistent with sarcoidosis found at autopsy in case 3, to our knowledge, this has not been reported before in Friedreich's ataxia and should be considered at the present time as a fortuitous finding. It is important to point out that, when sarcoidosis involves the myocardium, cardiac dysfunction not dissimilar to what is observed in Friedreich's ataxia can occur (Nissen and Berte, 1964).

On the basis of our studies, the sequence of morphological events in Friedreich's cardiomyopathy could be the following: possible initial hypertrophy, degeneration of the cardiac muscle cell, necrosis associated with a non-specific inflammatory infiltrate and replacement fibrosis.

The histopathological changes and their sequence observed in the heart have several features in common with the primary degenerative diseases of skeletal muscle, reinforcing the hypothesis that Friedreich's ataxia is a genetically determined degerative process simultaneously involving the nervous system and the heart.

Because experimental vitamin-E deficiency has been reported to produce necrosis, fibrotic replacement and pigment deposits in the posterior roots and columns in the nervous system (Malamud et al. 1949), the hypothesis of a metabolic defect related to this vitamin or other oligoelements should be entertained in Friedreich's ataxia. Although there is no evidence of a vitamin-E deficiency in patients suffering from Friedreich's ataxia, the decreased concentration of serum lipoprotein found in some patients (Davignon et al. 1979) could affect the transport of this particular vitamin (Horwitt et al. 1972, Kayden and Bjornson, 1972) and possibly produce a relative deficiency. This in turn, could have some repercussions on the biosynthesis of heme (Caasi et al. 1972) and could explain the increased free erythrocyte protoporphyrin levels recently detected in some patients with Friedreich's ataxia (Morgan et al. 1979).

The morphological studies of these 3 new cases do not support the views that a calcium disturbance is primarily related to Friedreich's cardiomyopathy and therefore, the Syrian hamster may not be an appropriate experimental model (Azari et al. 1979).

Further studies regarding the content of lipofuscin and trace metals particularly iron in the heart muscle are needed. An investigation is warranted of the antioxydative mechanisms which are vitamin-E-selenium dependent and which are essential for the metabolic and structural integrity of the plasma membrane.

\section{ACKNOWLEDGEMENTS}

We wish to acknowledge the technical assistance of Marcel Paquin and to thank Nicole Pichette and Donate Therrien for help in preparing the manuscript. We also thank Dr. Jean-Pierre Tremblay who provided us with the material from case 2 . These studies were funded by l'Association Canadienne de l'Ataxie de Friedreich.

\section{REFERENCES}

ADAMS R.A., DENNY-BROWN D. and PEARSON C.M. (1962): Diseases of muscle. A study in Pathology. Harper and Row, New York, pp. 268-282.

AZARI J., REISINE T., BARBEAU A., YAMAMURA H.I. and HUXTABLE R.: The Syrian Goden Hamster: A model for the Cardiomyopathy of Friedreich's ataxia (1979). Can. J. Neurol. Sci. 6: 223-226.

BOVE K.E.: Myocardial hypertrophy and enlargement (1974). In: The Heart. (Edwards J.E., Lev M. and Abell M.R., eds) Williams and Wilkins Co., Baltimore, International Academy of Pathology monograph no 12: pp. $30-55$. 
BUJA L.M. and ROBERTS W.C. (1971): Iron in the heart. Etiology and clinical significance. Amer. J. Med., 51: 209-221.

CASSI P.I., HAUSWIRTH J.W, and NAIR P.P. (1972): Biosynthesis of heme in vitamin E deficiency. Ann. N.Y. Acad. Sci. 203: 93102.

DAVIGNON J., HUANG Y.S., WOLF J.P. and BARBEAU A. (1979): Fatty acid profile of major lipid classes in plasma lipoproteins of patients with Friedreich's ataxia. Demonstration of a low linoleic acid content most evident in the cholesteryl-ester fraction. Can. J. Neurol. Sci., 6: 275-283.

GORE 1. and ARONS W., (1949): Calcification of the myocardium. A pathologic study of thirteen cases. Arch. Path., 48: 1-12.

HEGGTVEIT H.A. and NADKARNI B.B. (197I): Ultrastructural pathology of the myocardium. Meth. Achievm. exp. Path., 5: 474-517, ed. by E. Bajusz., Boston, Mass., and G. Jasmin, Montreal (Karger, Basel).

HEWER R.L., (1969): The heart in Friedreich's ataxia. Br. Heart J., 31: 5-14.

HORWITT M.K., HARVEY C.C., DAHM C.H. and SEARCY M.T., (1972): Relationship between tocopherol and serum lipid levels for determination of nutritional adequacy. Ann. N.Y. Acad. Sci., 203: 223236.

KAYDEN H.J. and BJORNSON L., (1972): The dynamics of vitamin $E$ transport in the human erythrocyte. Ann. N.Y. Acad. Sci., 203: 127-140.

KOOBS D.H., SCHULTZ R.L. and JUTZY R.V., (1978): The origin of lipofuscin and possible consequences to the myocardium. Arch. Pathol. Lab. Med., 102: 66-68.
MALAMUD N., NELSON M.M. and EVANS H.M., (1949): The effect of chronic vitamin E deficiency on the nervous system in the rat. Ann. N.Y. Acad. Sci., 52: 135-138.

MALKOFF D.B. and STREHLER B.L., (1963): The ultrastructure of isolated and in situ human cardiac age pigment. J. Cell. Biol., 16: 611-616.

MASON K.W. and EMMEL A.F. (1945): Vitamin $E$ and muscle pigment in the rat. Anat. Rec. 92: 33-60.

MORALES A.R., (1974): Cardiomyopathies: Congenital and acquired. In: The heart, (Edwards, J.E., Lev M. and Abel M.R., eds) Williams and Wilkins Co., Baltimore, International Academy of Pathology Monograph no 15: pp. 211-231.

MORGAN R.O., NAGLIE G, HOROBIN D.F. and BARBEAU A., (1979): Erythrocyte protoporphyrin levels in patients with Friedreich's and other ataxias. Can. J. Neurol. Sci., 6: 227-232.

NISHIOKA N., TAKAHATA N. and IIZUKA R., (1968): Histochemical studies on the lipopigments in the nerve cells. A comparison with lipofuscin and ceroid pigment. Acta Neuropath., 11: 174-181.

NISSEN A.W. and BERTE J.B., (1964): Cardiac arrhythmias in sarcoidosis. Arch. Int. Med., 113: 167-174.

PEARCE G.W. and WALTON J.N., (1962): Progressive muscular dystrophy: the histopathological changes in skeletal muscle obtained by biopsy. J. Path. Bact., 83: 535550.

PORTA E:A. and HARTROFT W.S., (1969): Lipid pigments in relation to aging and dietary factors (lipofuscins) In: Pigments in Pathology (Wolman M. ed.) Academic Press, New York, pp. 191-235.
REICHENBACH D.D. and BENDITT E.P., (1969): Myofibrillar degeneration: a common form of cardiac muscle injury. Ann. N.Y. Acad. Sci., 156: 164-176.

ROBERTS W.C., FERRANS V.J. and BUJA L.M., (1974): Pathologic aspects of the idiopathic cardiomyopathies. Comparative pathology of the heart. Adv. Cardiol., 13: 349-367 (Karger, Basel).

RUSSELL D.S., (1946): Myocarditis in Friedreich's ataxia. J. Path. Bact., 58: 739-748.

SANCHEZ-CASIS G., COTE $M$. and BARBEAU A., (1976): Pathology of the heart in Friedreich's ataxia: Review of the literature and report of one case. Can. J. Neurol. Sci., 3: 349-354.

SANDRITTER W, and SCOMAZZONI G., (1964): Deoxyribonucleic acid content (Feulgen photometry) and dry weight (interference microscopy) of normal and hypertrophic heart muscle fibres. Nature, 202: 100101.

SMITH E.R., SANGALANG V.E., HEFFERNAN L.P., WELCH J.P. and FLEMINGTON C.S., (1977): Hypertrophic cardiomyopathy: The heart disease of Friedreich's ataxia. Am. Heart J., 94: 428-434.

STREHLER B.L., MARK D.D., MILDVAN A.S. and GEE M.V., (1959): Rate and magnitude of age pigment accumulation in the human myocardium. J. Geront., 14: 430439.

WALTON J.N. and HUDGSON P., (1977): Inflammatory myopathies and related diseases. In: Scientific Approaches to Clinical Neurology, Vol. 2 (Goldensohn E.S. and Appel S.H., eds), Lea and Febiger, Philadelphia, pp. 1766-1779. 